QUARTERLY OF APPLIED MATHEMATICS

VOLUME LXX, NUMBER 2

JUNE 2012, PAGES 269-284

S 0033-569X(2012)01233-3

Article electronically published on February 3, 2012

\title{
CONTACT PROBLEMS FOR ELASTIC BODIES WITH RIGID INCLUSIONS
}

\author{
BY \\ ALEXANDER KHLUDNEV \\ Lavrentyev Institute of Hydrodynamics of the Russian Academy of Sciences, and Novosibirsk State \\ University, Novosibirsk 630090, Russia
}

\begin{abstract}
This paper is concerned with a new type of free boundary problems for elastic bodies with a rigid inclusion being in contact with another rigid inclusion or with a non-deformable punch. We propose correct problem formulations with inequality type boundary conditions of a non-local type describing a mutual non-penetration between surfaces. Solution existence is proved for different types of inclusions and different geometries. Qualitative properties of solutions are analyzed provided that rigidity parameters are changed.
\end{abstract}

1. Introduction. Contact problems of elasticity theory with unknown contact sets are characterized by non-linear boundary conditions. In the framework of this approach we can mention the Signorini problems [1, [6], contact problems for bodies of different dimensions [4], 12], 13], and crack problems with unknown contact sets [3], 8], 9], 10], [15. For each class of these problems, boundary conditions are of inequality type, and a contact set is determined provided that a solution of the problem is found. Therefore, the problems analyzed can be considered as free boundary problems. In recent years a new type of problems has been under active research. We have in mind elastic bodies with rigid inclusions and cracks located at interfaces between elastic and rigid parts. We refer the reader to the works [2, [5], [7, [13, [16], [17, [18, [19], where correct formulations and results for elastic bodies with rigid inclusions and cracks can be found. Such problems are of great interest due to the active study of composite materials. Modeling of composites takes into account different types of inclusions and cracks of complicated geometries. Energy release through crack propagation in brittle composites is a subject of many current investigations. Resistance against damage and failure is the driving motivation of the mathematical analysis. In material sciences one intends to improve a quality using properties of rigid fibres and other inclusions. Practical examples and numerics for composite materials can be found, for instance, in [11], [14].

Received May 24, 2010.

2010 Mathematics Subject Classification. Primary 35J20, 74E30.

Key words and phrases. Rigid inclusion, non-penetration condition, delamination, crack.

E-mail address: khlud@hydro.nsc.ru

(C) 2012 Brown University 
There is one more class of contact problems with unknown boundaries. Indeed, new types of problems appear provided that we have two or more rigid inclusions with the non-penetration condition of inequality type between their surfaces. It is known that a stress tensor is not defined in a rigid part of the body, and there is no possibility to describe boundary conditions in terms of stress tensor components. Hence, this class of free boundary problems requires a suitable problem formulation and investigation.

In this paper, we consider a wide class of equilibrium problems for elastic bodies containing two rigid inclusions or a rigid inclusion being in contact with a non-deformable punch. We propose correct problem formulations from the standpoint of mechanics. Both volume and thin rigid inclusions are analyzed. Differential and variational problem formulations are discussed, and a solution existence is proved. Fictitious domain approach is applied to establish relations between different types of problems. Other qualitative properties of solutions are investigated, in particular, a passage to a limit is justified provided that a rigidity parameter goes to infinity.

2. Rigid inclusion being in a contact with non-deformable punch on the boundary. Let $\Omega \subset R^{2}$ be a bounded domain with smooth boundary $\Gamma$, and $\omega \subset R^{2}$ be a subdomain with a location depicted in Fig. 1. We assume that the boundary $\partial \omega$ of $\omega$ is Lipschitz, $\gamma=\Gamma \cap \partial \omega, \partial \omega=S \cup \gamma, \Gamma=\Gamma_{0} \cup \Sigma \cup \gamma$, and meas $(\Gamma \cap \partial \omega)>0$. The domain $\Omega \backslash \bar{\omega}$ corresponds to an elastic part of the body, and $\omega$ fits to a rigid part. On the part $\gamma$ of the boundary $\Gamma$ we consider a contact between the rigid part $\omega$ and a non-deformable punch (which is situated outside of $\Omega$ ). Denote by $n=\left(n_{1}, n_{2}\right), \nu=\left(\nu_{1}, \nu_{2}\right)$ unit normal vectors to $\Gamma$ and to $\partial \omega$, respectively.

To provide a problem formulation we assume that $f=\left(f_{1}, f_{2}\right) \in L_{\text {loc }}^{2}\left(R^{2}\right)$ is given, and the elasticity tensor $A=\left\{a_{i j k l}\right\} \in L_{l o c}^{\infty}\left(R^{2}\right)$ is also given, with the usual properties of symmetry and positive definiteness:

$$
\begin{array}{r}
a_{i j k l}=a_{j i k l}=a_{k l i j}, \quad i, j, k, l=1,2, \\
a_{i j k l} \xi_{i j} \xi_{k l} \geq c_{0}|\xi|^{2} \quad \forall \xi_{j i}=\xi_{i j}, c_{0}>0 .
\end{array}
$$

We accept a summation convention over repeated indices; all values with two subindices are assumed to be symmetric in those indices.

Introduce the set of admissible displacements

$$
K=\left\{v=\left(v_{1}, v_{2}\right) \in H_{\Gamma_{0}}^{1}(\Omega) \mid v \nu \geq 0 \text { on } \gamma,\left.v\right|_{\omega} \in R(\omega)\right\},
$$

where

$$
H_{\Gamma_{0}}^{1}(\Omega)=\left\{v \in H^{1}(\Omega) \mid v=0 \text { on } \Gamma_{0}\right\},
$$

and the space of infinitesimal rigid displacements $R(\omega)$ is defined as follows:

$$
R(\omega)=\left\{\rho=\left(\rho_{1}, \rho_{2}\right) \mid \rho(x)=B x+C, x \in \omega\right\}
$$

with

$$
B=\left(\begin{array}{rr}
0 & b \\
-b & 0
\end{array}\right), C=\left(c^{1}, c^{2}\right) ; b, c^{1}, c^{2}=\text { const }
$$




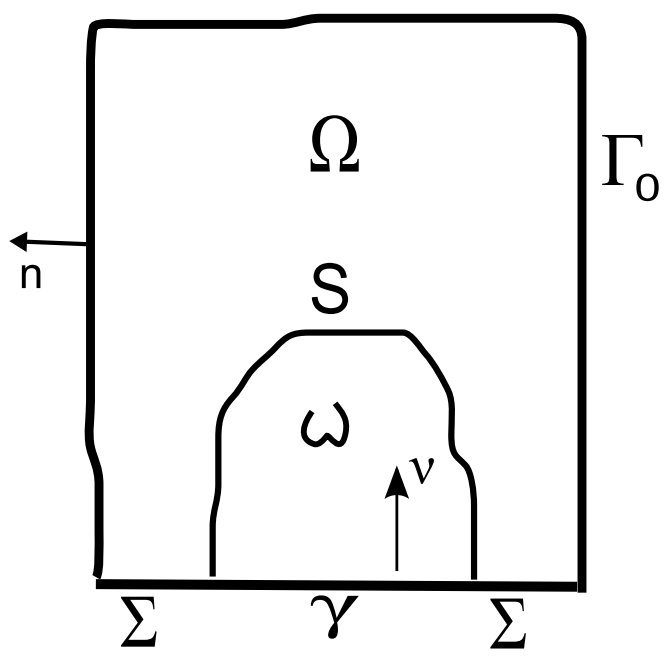

FIG. 1

Now we are in a position to formulate an equilibrium problem for the body with elastic and rigid parts $\Omega \backslash \bar{\omega}$ and $\omega$, respectively, where the rigid part is in contact with the nondeformable punch on $\gamma$. Namely, it is necessary to find functions $u=\left(u_{1}, u_{2}\right), \rho_{0} \in$ $R(\omega), \sigma=\left\{\sigma_{i j}\right\}, i, j=1,2$, such that

$$
\begin{array}{r}
-\operatorname{div} \sigma=f \quad \text { in } \quad \Omega \backslash \bar{\omega}, \\
\sigma-A \varepsilon(u)=0 \quad \text { in } \quad \Omega \backslash \bar{\omega}, \\
u=0 \quad \text { on } \quad \Gamma_{0}, \sigma n=0 \quad \text { on } \quad \Sigma, \\
u=\rho_{0} \quad \text { on } \omega, \rho_{0} \nu \geq 0 \quad \text { on } \quad \gamma, \\
\int_{S} \sigma \nu \cdot \rho_{0}=\int_{\omega} f \rho_{0}, \\
\int_{S} \sigma \nu \cdot v \geq \int_{\omega} f v \quad \forall v \in K .
\end{array}
$$

Here (11) are equilibrium equations, and (2) is Hooke's law; $u=\left(u_{1}, u_{2}\right)$ is a displacement vector; $\sigma=\left\{\sigma_{i j}\right\}$ is a stress tensor; $\varepsilon(u)=\left\{\varepsilon_{i j}(u)\right\}$ is a strain tensor, $\varepsilon_{i j}(u)=\frac{1}{2}\left(u_{i, j}+\right.$ $\left.u_{j, i}\right), i, j=1,2, \sigma \nu=\left(\sigma_{1 j} \nu_{j}, \sigma_{2 j} \nu_{j}\right)$. Note that the stress tensor is not defined in the rigid part $\omega$. The second inequality of (4) describes a non-penetration between the rigid part and the non-deformable punch.

Along with the differential formulation of the problem (1)-(6) we provide a variational approach. To this end, consider the energy functional

$$
\Pi(v)=\frac{1}{2} \int_{\Omega \backslash \bar{\omega}} \sigma(v) \varepsilon(v)-\int_{\Omega} f v .
$$


To simplify the notation, we write $\sigma(v) \varepsilon(v)$ instead of $\sigma_{i j}(v) \varepsilon_{i j}(v)$, and $f v$ instead of $f_{i} v_{i} ; \sigma(v)=A \varepsilon(v)$. It is possible to prove that the minimization problem

$$
\inf _{v \in K} \Pi(v)
$$

has (a unique) solution satisfying the variational inequality

$$
u \in K, \quad \int_{\Omega \backslash \bar{\omega}} \sigma(u) \varepsilon(\bar{u}-u) \geq \int_{\Omega} f(\bar{u}-u) \quad \forall \bar{u} \in K .
$$

Indeed, the functional $\Pi$ is coercive on $K$ and weakly lower semicontinuous on $H_{\Gamma_{0}}^{1}(\Omega)$.

Now we prove that problem formulations (1) -(6) and (8) are equivalent, assuming that the solution is smooth enough. Let $u=\rho_{0} \in R(\omega)$ on $\omega$. We choose $\bar{u}=u \pm \varphi$ in (8),$\varphi=\left(\varphi_{1}, \varphi_{2}\right) \in C_{0}^{\infty}(\Omega \backslash \bar{\omega})$, and make an extension of $\varphi$ by zero in $\omega$. Then $\bar{u} \in K$, and (8) implies that

$$
\int_{\Omega \backslash \bar{\omega}} \sigma(u) \varepsilon(\varphi)=\int_{\Omega \backslash \bar{\omega}} f \varphi \quad \forall \varphi=\left(\varphi_{1}, \varphi_{2}\right) \in C_{0}^{\infty}(\Omega \backslash \bar{\omega}),
$$

which provides a fulfillment of (11) in the distributional sense. If we choose $\bar{u}=u \pm \psi$ in (8), $\psi=\left(\psi_{1}, \psi_{2}\right)$ an arbitrary smooth function, $\operatorname{supp} \psi \in \bar{B}_{x_{0}}$, where $x_{0} \in \Sigma$, and $B_{x_{0}}$ is a neighborhood of $x_{0}$, the following relation is obtained:

$$
\pm \int_{\Omega \backslash \bar{\omega}} \sigma(u) \varepsilon(\psi) \geq \pm \int_{\Omega \backslash \bar{\omega}} f \psi
$$

and therefore

$$
\int_{\Sigma} \sigma(u) n \cdot \psi=0
$$

which implies the second relation of (3). Now take $\bar{u}=0, \bar{u}=2 u$ in (8). This yields

$$
\int_{\Omega \backslash \bar{\omega}} \sigma(u) \varepsilon(u)=\int_{\Omega} f u .
$$

Integrating by parts here, in view of (1), we arrive at the relation

$$
\int_{S} \sigma(u) \nu \cdot u=\int_{\omega} f u
$$

i.e., (5) follows. Choosing $\bar{u}=u+v$ in (8), $v \in K$, we obtain

$$
\int_{\Omega \backslash \bar{\omega}} \sigma(u) \varepsilon(v) \geq \int_{\Omega} f v \quad \forall v \in K
$$

hence, again after integration by parts,

$$
\int_{S} \sigma(u) \nu \cdot v \geq \int_{\omega} f v \quad \forall v \in K
$$

Thus (6) follows.

All the remaining relations of (11)-(6) hold by the definition of the solution to (8). Hence we prove that (11)-(6) follow from (8). Conversely, (1) implies that

$$
\int_{\Omega \backslash \bar{\omega}}(-\operatorname{div} \sigma-f)(\bar{u}-u)=0 \quad \forall \bar{u} \in K .
$$


Integration by parts in this relation provides, by (3),

$$
\int_{\Omega \backslash \bar{\omega}} \sigma(u) \varepsilon(\bar{u}-u)-\int_{\Omega \backslash \bar{\omega}} f(\bar{u}-u)=\int_{S} \sigma(u) \nu \cdot(\bar{u}-u) .
$$

We know that $\bar{u}=\rho \in R(\omega), u=\rho_{0}$ on $S$; hence, by (5), (6),

$$
\int_{S} \sigma(u) \nu \cdot\left(\rho-\rho_{0}\right) \geq \int_{\omega} f \rho-\int_{\omega} f \rho_{0}
$$

and consequently, (8) is obtained.

In what follows we prove that the problem (11)-(6) can be obtained as a limit of the Signorini type problems considered in the domain $\Omega$. In fact, introduce a family $A^{\lambda}=\left\{a_{i j k l}^{\lambda}\right\}$ of elasticity tensors defined in $\Omega$,

$$
a_{i j k l}^{\lambda}=\left\{\begin{array}{ccc}
a_{i j k l} & \text { in } & \Omega \backslash \bar{\omega} \\
\lambda^{-1} a_{i j k l} & \text { in } & \omega
\end{array}\right.
$$

with a positive parameter $\lambda$, and consider a family of the Signorini problems: find functions $u^{\lambda}=\left(u_{1}^{\lambda}, u_{2}^{\lambda}\right), \sigma^{\lambda}=\left\{\sigma_{i j}^{\lambda}\right\}, i, j=1,2$, such that

$$
\begin{array}{r}
-\operatorname{div} \sigma^{\lambda}=f \quad \text { in } \quad \Omega, \\
\sigma^{\lambda}-A^{\lambda} \varepsilon\left(u^{\lambda}\right)=0 \quad \text { in } \quad \Omega, \\
u^{\lambda}=0 \quad \text { on } \quad \Gamma_{0}, \sigma^{\lambda} n=0 \quad \text { on } \quad \Sigma, \\
u^{\lambda} n \leq 0 \quad \text { on } \quad \gamma, \\
\int_{\gamma} \sigma^{\lambda} n \cdot u^{\lambda}=0, \\
\int_{\gamma} \sigma^{\lambda} n \cdot v \geq 0 \quad \forall v \in K_{c},
\end{array}
$$

where

$$
K_{c}=\left\{v=\left(v_{1}, v_{2}\right) \in H_{\Gamma_{0}}^{1}(\Omega) \mid v n \leq 0 \text { on } \gamma\right\} .
$$

The problem (10)-(15) is solvable, and the solution existence can be proved by minimization of the energy functional

$$
\frac{1}{2} \int_{\Omega} \sigma^{\lambda}(v) \varepsilon(v)-\int_{\Omega} f v
$$

over the set $K_{c}$. This minimization problem has a solution, and the solution satisfies the variational inequality

$$
u^{\lambda} \in K_{c}, \quad \int_{\Omega} \sigma^{\lambda}\left(u^{\lambda}\right) \varepsilon\left(\bar{u}-u^{\lambda}\right) \geq \int_{\Omega} f\left(\bar{u}-u^{\lambda}\right) \quad \forall \bar{u} \in K_{c} .
$$

It is possible to derive a priori estimates for $u^{\lambda}$. To this end, we substitute $\bar{u}=0, \bar{u}=$ $2 u^{\lambda}$ in (16) as test functions. This implies that

$$
\int_{\Omega \backslash \bar{\omega}} \sigma\left(u^{\lambda}\right) \varepsilon\left(u^{\lambda}\right)+\frac{1}{\lambda} \int_{\omega} \sigma\left(u^{\lambda}\right) \varepsilon\left(u^{\lambda}\right)=\int_{\Omega} f u^{\lambda} .
$$


The relation obtained implies, uniformly in $0<\lambda<\lambda_{0}$,

$$
\left\|u^{\lambda}\right\|_{H_{\Gamma_{0}}^{1}(\Omega)} \leq c, \frac{1}{\lambda} \int_{\omega} \sigma\left(u^{\lambda}\right) \varepsilon\left(u^{\lambda}\right) \leq c \lambda
$$

with a positive constant $c$. We can choose a subsequence, with the previous notation, such that as $\lambda \rightarrow 0$,

$$
u^{\lambda} \rightarrow u \text { weakly in } H_{\Gamma_{0}}^{1}(\Omega) ; u=\rho_{0} \text { on } \omega, \rho_{0} \in R(\omega) .
$$

By this convergence, it is possible to pass to the limit in (16) as $\lambda \rightarrow 0$. Any element $\bar{u} \in K$ satisfies the inclusion $u \in K_{c}$. Substituting $\bar{u} \in K$ in (16) we derive

$$
\int_{\Omega \backslash \bar{\omega}} \sigma\left(u^{\lambda}\right) \varepsilon\left(\bar{u}-u^{\lambda}\right)+\frac{1}{\lambda} \int_{\omega} \sigma\left(u^{\lambda}\right) \varepsilon\left(-u^{\lambda}\right) \geq \int_{\Omega} f\left(\bar{u}-u^{\lambda}\right) .
$$

The passage to the limit here as $\lambda \rightarrow 0$ provides the relation

$$
u \in K, \quad \int_{\Omega \backslash \bar{\omega}} \sigma(u) \varepsilon(\bar{u}-u) \geq \int_{\Omega} f(\bar{u}-u) \quad \forall \bar{u} \in K,
$$

i.e. the limit function from (17) solves the problem (10)-(6).

3. Thin rigid inclusion on the contact boundary. Let $\Omega \subset R^{2}$ be a bounded domain with smooth boundary $\Gamma$. As in the previous section, $\Gamma=\Gamma_{0} \cup \Sigma \cup \gamma$, meas $\gamma$ $>0$. Assume that $\gamma$ represents a thin rigid inclusion (located on the boundary $\Gamma$ ) which is in contact with a non-deformable punch; see Fig. 2. Let $\nu=\left(\nu_{1}, \nu_{2}\right)$ be a unit normal internal vector to $\Gamma$. Introduce a notation for the set of admissible displacements

$$
K_{0}=\left\{v=\left(v_{1}, v_{2}\right) \in H_{\Gamma_{0}}^{1}(\Omega) \mid v \nu \geq 0 \text { on } \gamma,\left.v\right|_{\gamma} \in R(\gamma)\right\},
$$

where

$$
R(\gamma)=\left\{\rho=\left(\rho_{1}, \rho_{2}\right) \mid \rho(x)=B x+C, x \in \gamma\right\}
$$

with

$$
B=\left(\begin{array}{rr}
0 & b \\
-b & 0
\end{array}\right), C=\left(c^{1}, c^{2}\right) ; b, c^{1}, c^{2}=\text { const } .
$$

Formulation of an equilibrium problem for the body $\Omega$ with the thin rigid inclusion $\gamma$ is as follows. We have to find functions $u=\left(u_{1}, u_{2}\right), \rho_{0} \in R(\gamma), \sigma=\left\{\sigma_{i j}\right\}, i, j=1,2$, such that

$$
\begin{array}{r}
-\operatorname{div} \sigma=f \quad \text { in } \quad \Omega, \\
\sigma-A \varepsilon(u)=0 \quad \text { in } \quad \Omega, \\
u=0 \quad \text { on } \quad \Gamma_{0}, \sigma \nu=0 \quad \text { on } \quad \Sigma, \\
u=\rho_{0} \quad \text { on } \gamma, \rho_{0} \nu \geq 0 \text { on } \quad \gamma, \\
\int_{\gamma} \sigma \nu \cdot \rho_{0}=0, \\
\int_{\gamma} \sigma \nu \cdot v \leq 0 \quad \forall v \in K_{0} .
\end{array}
$$




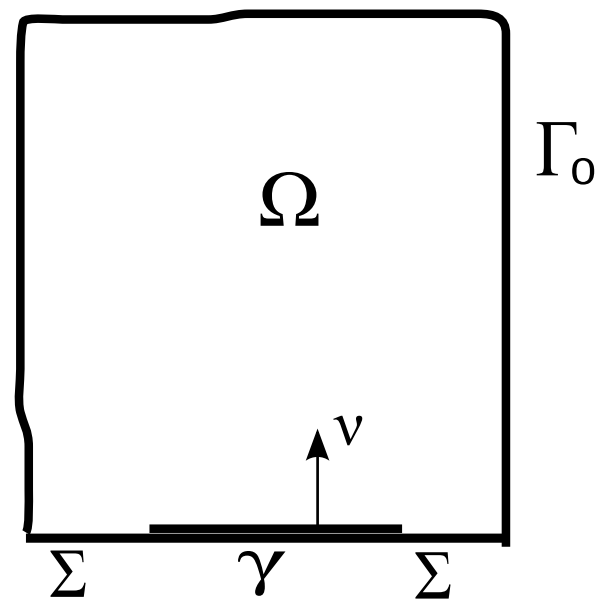

FIG. 2

It is possible to provide a variational formulation of the problem (19)-(24). Indeed, we have to find a solution of the minimization problem

$$
\inf _{v \in K_{0}}\left\{\frac{1}{2} \int_{\Omega} \sigma(v) \varepsilon(v)-\int_{\Omega} f v\right\} .
$$

A solution of this problem exists and satisfies the variational inequality

$$
u \in K_{0}, \quad \int_{\Omega} \sigma(u) \varepsilon(\bar{u}-u) \geq \int_{\Omega} f(\bar{u}-u) \quad \forall \bar{u} \in K_{0} .
$$

Below we state that problem formulations (19)-(24) and (25) are equivalent. It is clear that (19) holds, and it follows from (25). Substituting $\bar{u}=0, \bar{u}=2 u$ in (25) we derive

$$
\int_{\Omega} \sigma(u) \varepsilon(u)=\int_{\Omega} f u .
$$

Since $u=0$ on $\Gamma_{0}$, and $u=\rho_{0}$ on $\gamma$, it follows from (26) that

$$
\int_{\Sigma} \sigma \nu \cdot u+\int_{\gamma} \sigma \nu \cdot \rho_{0}=0
$$

Meanwhile a substitution of $\bar{u}=u \pm \varphi$ in (25), $\varphi=\left(\varphi_{1}, \varphi_{2}\right)$ a smooth function, where $B_{x_{0}}$ is a neighborhood of a point $x_{0} \in \operatorname{int} \Sigma, \operatorname{supp} \varphi \in B_{x_{0}}$, provides the relation

$$
\int_{\Omega} \sigma(u) \varepsilon(\varphi)=\int_{\Omega} f \varphi,
$$

whence the second condition of (21) follows. Thus (27) implies (23). Since $\bar{u}=u+v \in K_{0}$, where $v \in K_{0}$, the variational inequality (25) implies that

$$
\int_{\Omega} \sigma(u) \varepsilon(v) \geq \int_{\Omega} f v
$$

and hence, (24) is derived. 


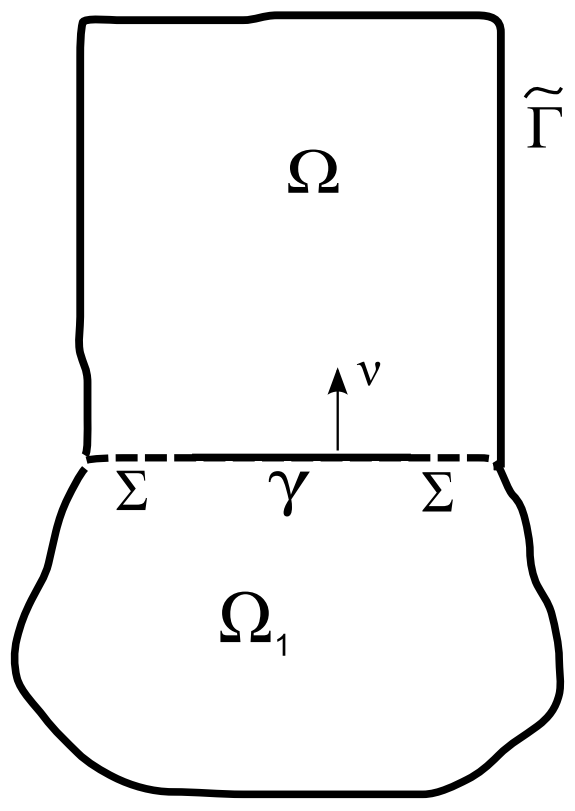

FIG. 3

Conversely, let (19)-(24) be fulfilled. Multiply (19) by $\bar{u}-u, \bar{u} \in K_{0}$. This gives

$$
\int_{\Omega}(-\operatorname{div} \sigma-f)(\bar{u}-u)=0
$$

and thus

$$
\int_{\Omega} \sigma(u) \varepsilon(\bar{u}-u)-\int_{\Omega} f(\bar{u}-u)+\int_{\gamma} \sigma(u) \nu \cdot(\bar{u}-u)=0 .
$$

To prove that (25) takes place it suffices to establish the inequality

$$
\int_{\gamma} \sigma(u) \nu \cdot(\bar{u}-u) \leq 0 .
$$

But this relation follows directly from (23), (24).

It turns out that the problem (25) can be considered as a limit of crack type problems. Indeed, consider a family of boundary value problems describing an equilibrium of an elastic body with a delaminated thin rigid inclusion. To describe this approach, we introduce a fictitious domain $\Omega_{1}$ as shown in Fig. 3. Denote $\tilde{\Gamma}=\Gamma_{0} \cup\left(\partial \Omega_{1} \backslash(\gamma \cup \Sigma)\right)$, and let $A^{\lambda}=\left\{a_{i j k l}^{\lambda}\right\}$,

$$
a_{i j k l}^{\lambda}=\left\{\begin{array}{cl}
a_{i j k l} & \text { in } \Omega, \\
\lambda^{-1} a_{i j k l} & \text { in } \quad \Omega_{1}, \lambda>0 .
\end{array}\right.
$$

We introduce next a set of admissible displacements

$$
K_{s}=\left\{v=\left(v_{1}, v_{2}\right) \in H_{\tilde{\Gamma}}^{1}\left(\Omega \cup \Omega_{1}\right) \mid[v] \nu \geq 0 \text { on } \gamma,\left.v\right|_{\gamma^{+}} \in R(\gamma)\right\},
$$

where $[v]=v^{+}-v^{-}$is a jump of the vector $v$ on $\gamma$. 
Then the equilibrium problem to be considered is as follows. We have to find functions $u^{\lambda}=\left(u_{1}^{\lambda}, u_{2}^{\lambda}\right), \rho_{0}^{\lambda} \in R(\gamma), \sigma^{\lambda}=\left\{\sigma_{i j}^{\lambda}\right\}, i, j=1,2$, such that

$$
\begin{array}{r}
-\operatorname{div} \sigma^{\lambda}=f \quad \text { in } \quad \Omega \cup \Omega_{1}, \\
\sigma^{\lambda}-A^{\lambda} \varepsilon\left(u^{\lambda}\right)=0 \quad \text { in } \quad \Omega \cup \Omega_{1}, \\
u^{\lambda}=0 \quad \text { on } \quad \tilde{\Gamma}, \sigma^{\lambda} \nu=0 \quad \text { on } \quad \Sigma^{ \pm}, \\
{\left[u^{\lambda}\right] \nu \geq 0 \quad \text { on } \quad \gamma, u^{\lambda}=\rho_{0}^{\lambda} \quad \text { on } \quad \gamma^{+},} \\
\int_{\gamma}\left[\sigma^{\lambda} \nu \cdot u^{\lambda}\right]=0, \\
\int_{\gamma}\left[\sigma^{\lambda} \nu \cdot v\right] \leq 0 \quad \forall v \in K_{s} .
\end{array}
$$

Solution existence of the problem (29)-(34) can be proved from the variational inequality

$$
u \in K_{s}, \quad \int_{\Omega \cup \Omega_{1}} \sigma^{\lambda}\left(u^{\lambda}\right) \varepsilon\left(\bar{u}-u^{\lambda}\right) \geq \int_{\Omega \cup \Omega_{1}} f\left(\bar{u}-u^{\lambda}\right) \quad \forall \bar{u} \in K_{s} .
$$

We should remark that (29)-(34) and (35) are equivalent in the sense that (35) follows from (29)-(34), and conversely, (29)-(34) follow from (35), provided that the solution is smooth. Now we justify a passage to the limit as $\lambda \rightarrow 0$ in (33). From (35) we obtain

$$
\int_{\Omega} \sigma\left(u^{\lambda}\right) \varepsilon\left(u^{\lambda}\right)+\frac{1}{\lambda} \int_{\Omega_{1}} \sigma\left(u^{\lambda}\right) \varepsilon\left(u^{\lambda}\right)=\int_{\Omega \cup \Omega_{1}} f u^{\lambda},
$$

and hence uniformly in $\lambda \in\left(0, \lambda_{0}\right)$,

$$
\left\|u^{\lambda}\right\|_{H_{\tilde{\Gamma}}^{1}\left(\Omega \cup \Omega_{1}\right)} \leq c,\left\|u^{\lambda}\right\|_{H^{1}\left(\Omega_{1}\right)}^{2} \leq c \lambda .
$$

Choosing a subsequence, if necessary, we assume that as $\lambda \rightarrow 0$,

$$
u^{\lambda} \rightarrow u \text { weakly in } H_{\tilde{\Gamma}}^{1}\left(\Omega \cup \Omega_{1}\right) ; u^{\lambda} \rightarrow 0 \text { strongly in } H^{1}\left(\Omega_{1}\right) .
$$

Now, it is possible to substitute in (35) a test function $\bar{u} \in K_{s}$ such that $\bar{u}=0$ in $\Omega_{1}$, $\bar{u}=\bar{\rho}$ on $\gamma^{+}, \bar{\rho} \nu \geq 0$ on $\gamma^{+}$. Note that a restriction of $\bar{u}$ to $\Omega$ belongs to the set $K_{0}$. In such a case a passage to the limit as $\lambda \rightarrow 0$ in (35) provides the variational inequality

$$
u \in K_{0}, \quad \int_{\Omega} \sigma(u) \varepsilon(\bar{u}-u) \geq \int_{\Omega} f(\bar{u}-u) \quad \forall \bar{u} \in K_{0}
$$

coinciding with (25), which is what is needed.

4. Thin rigid inclusion contiguous to volume rigid inclusion. Let $\Omega \subset R^{2}$ be a bounded domain with smooth boundary $\Gamma$, and $\omega \subset \Omega$ be a subdomain with smooth boundary $\partial \omega, \partial \omega \cap \Gamma=\emptyset$. Assume that $\gamma$ is a smooth curve without self-intersections, $\gamma \cap \Gamma \neq \emptyset$, and $\partial \omega \cap \gamma=\left\{x_{0}\right\}$. In our considerations, $\omega$ corresponds to a volume rigid inclusion, and $\gamma$ fits to a thin rigid inclusion, $\Omega_{\gamma}=\Omega \backslash \bar{\gamma}$. Let $n=\left(n_{1}, n_{2}\right), \nu=\left(\nu_{1}, \nu_{2}\right)$ be unit normal vectors to $\partial \omega$ and to $\gamma$, respectively; see Fig. 4 . 


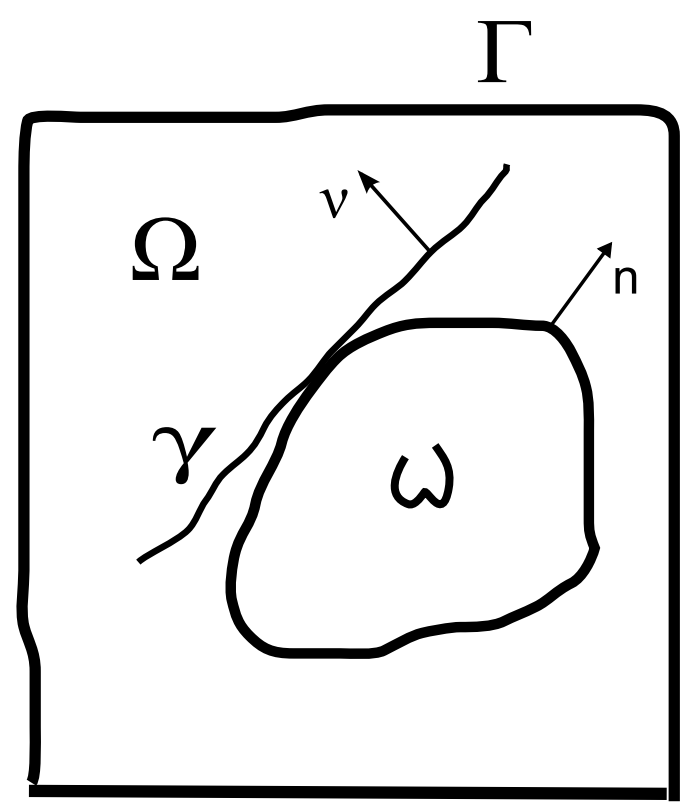

FIG. 4

We assume that a delamination takes place at $\gamma^{-}$; hence we should consider a crack at $\gamma^{-}$. The problem formulation is as follows. It is necessary to find functions $u=$ $\left(u_{1}, u_{2}\right), \rho_{0} \in R(\gamma), \rho_{1} \in R(\omega), \sigma=\left\{\sigma_{i j}\right\}, i, j=1,2$, such that

$$
\begin{array}{r}
-\operatorname{div} \sigma=f \quad \text { in } \quad \Omega_{\gamma} \backslash \bar{\omega}, \\
\sigma-A \varepsilon(u)=0 \quad \text { in } \quad \Omega_{\gamma} \backslash \bar{\omega}, \\
u=0 \quad \text { on } \quad \Gamma,[u] \nu \geq 0 \quad \text { on } \gamma, \\
u=\rho_{0} \quad \text { on } \gamma^{+}, u=\rho_{1} \quad \text { on } \omega, \\
-\int_{\gamma}[\sigma \nu \cdot u]-\int_{\partial \omega} \sigma n \cdot u=\int_{\omega} f u, \\
-\int_{\gamma}[\sigma \nu \cdot \tilde{u}]-\int_{\partial \omega} \sigma n \cdot \tilde{u} \geq \int_{\omega} f \tilde{u} \quad \forall \tilde{u} \in K_{1} .
\end{array}
$$

Here the set of admissible displacements is defined as follows:

$$
\begin{array}{r}
K_{1}=\left\{v=\left(v_{1}, v_{2}\right) \in H_{\Gamma}^{1}\left(\Omega_{\gamma}\right) \mid[v] \nu \geq 0 \text { on } \gamma,\right. \\
\left.\left.v\right|_{\omega} \in R(\omega),\left.v\right|_{\gamma^{+}} \in R(\gamma)\right\} .
\end{array}
$$

We can prove a solvability of the problem (36)-(41). It can be stated by a variational method. Indeed, the problem

$$
\inf _{v \in K_{1}}\left\{\frac{1}{2} \int_{\Omega_{\gamma} \backslash \bar{\omega}} \sigma(v) \varepsilon(v)-\int_{\Omega_{\gamma}} f v\right\}
$$


has a solution $u$ satisfying the variational inequality

$$
u \in K_{1}, \quad \int_{\Omega_{\gamma} \backslash \bar{\omega}} \sigma(u) \varepsilon(\bar{u}-u) \geq \int_{\Omega_{\gamma}} f(\bar{u}-u) \quad \forall \bar{u} \in K_{1} .
$$

Let us check that problem formulations (36)-(41) and (42) are equivalent. Substituting $\bar{u}=0, \bar{u}=2 u$ in (42) as test functions we find

$$
\int_{\Omega_{\gamma} \backslash \bar{\omega}} \sigma(u) \varepsilon(u)=\int_{\Omega_{\gamma}} f u .
$$

Integrating by parts here we arrive at (40). On the other hand, $\bar{u}=u+\tilde{u} \in K_{1}$, where $\tilde{u} \in K_{1}$; hence from (42) it follows that

$$
\int_{\Omega_{\gamma} \backslash \bar{\omega}} \sigma(u) \varepsilon(\tilde{u}) \geq \int_{\Omega_{\gamma}} f \tilde{u} \quad \forall \tilde{u} \in K_{1}
$$

thus (41) follows. All the remaining relations of (36)-(41) are obviously included in (42). Conversely, multiplying (36) by $\bar{u}-u$, where $\bar{u} \in K_{1}$, and integrating over $\Omega_{\gamma} \backslash \bar{\omega}$ we can obtain (42), which is what is needed.

5. Thin rigid inclusion being in contact with volume rigid inclusion. Let $\Omega \subset R^{2}$ be a bounded domain with smooth boundary $\Gamma$, and $\omega \subset \Omega$ be a subdomain with smooth boundary $\partial \omega ; \partial \omega \cap \Gamma=\emptyset$. Also, let $\gamma \subset \Omega$ be a smooth curve without self-intersections such that its part $\gamma_{0}$ coincides with a part of the boundary $\partial \omega$; see Fig. 5. Denote by $n=\left(n_{1}, n_{2}\right), \nu=\left(\nu_{1}, \nu_{2}\right)$ unit normal vectors to $\partial \omega$ and $\gamma$, respectively. The domain $\omega$ fits to a volume rigid inclusion, and $\gamma$ represents a thin rigid inclusion, $S=\partial \omega \backslash \gamma_{0}$. In what follows we assume that a delamination takes place on $\gamma^{-}$; hence the non-penetration condition should be fulfilled on $\gamma_{0}$, as well as on $\gamma \backslash \gamma_{0}$.

The problem formulation is as follows. We have to find functions $u=\left(u_{1}, u_{2}\right), \rho_{0} \in$ $R(\gamma), \rho_{1} \in R(\omega), \sigma=\left\{\sigma_{i j}\right\}, i, j=1,2$, such that

$$
\begin{array}{r}
-\operatorname{div} \sigma=f \quad \text { in } \Omega_{\gamma} \backslash \bar{\omega}, \\
\sigma-A \varepsilon(u)=0 \quad \text { in } \Omega_{\gamma} \backslash \bar{\omega}, \\
u=0 \text { on } \Gamma,[u] \nu \geq 0 \text { on } \gamma, \\
u=\rho_{0} \text { on } \gamma^{+}, u=\rho_{1} \quad \text { on } \omega, \\
-\int_{\gamma \backslash \gamma_{0}}[\sigma \nu \cdot u]-\int_{\gamma_{0}} \sigma \nu \cdot u-\int_{S} \sigma n \cdot u=\int_{\omega} f u, \\
-\int_{\gamma \backslash \gamma_{0}}[\sigma \nu \cdot \tilde{u}]-\int_{\gamma_{0}} \sigma \nu \cdot \tilde{u}-\int_{S} \sigma n \cdot \tilde{u} \geq \int_{\omega} f \tilde{u} \quad \forall \tilde{u} \in K_{1} .
\end{array}
$$

The set of admissible displacements $K_{1}$ was defined in the previous section. Despite a different geometry (cf. Fig. 4), its formal definition is the same.

Note that the values $\sigma n$ in (47)-(48) are taken, in fact, on $S^{+}$; the values $\sigma \nu \cdot u$ and $\sigma \nu \cdot \tilde{u}$ in (47)-(48), included in the integrals over $\gamma_{0}$, are taken on $\gamma_{0}^{+}$.

Variational formulation of the problem (43)-(48) is as follows:

$$
u \in K_{1}, \quad \int_{\Omega_{\gamma} \backslash \bar{\omega}} \sigma(u) \varepsilon(\bar{u}-u) \geq \int_{\Omega_{\gamma}} f(\bar{u}-u) \quad \forall \bar{u} \in K_{1} .
$$




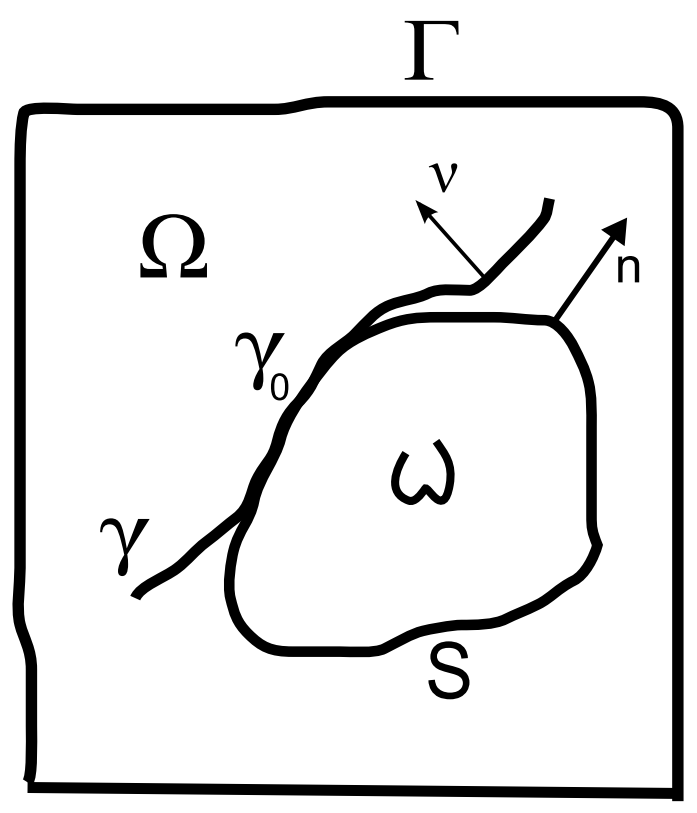

FIG. 5

We can prove the solution existence to the problem (49), and we can verify that problem formulations (43)-(48) and (49) are equivalent.

6. Thin rigid inclusion located inside the domain and on the boundary. Let $\Omega \subset R^{2}$ be a bounded domain with smooth boundary $\Gamma$, and $\gamma=\gamma_{1} \cup \gamma_{2}$ be a smooth curve such that its part $\gamma_{1}$ belongs to $\Gamma$, and $\gamma_{2} \subset \Omega$; see Fig. 6 . Denote by $\nu=\left(\nu_{1}, \nu_{2}\right)$ a unit normal vector to $\gamma$.

For simplicity we suppose that $\gamma_{1}$ and $\gamma_{2}$ are smooth curves. Denote $\Gamma_{0}=\Gamma \backslash \gamma_{1}, \Omega_{\gamma_{2}}=$ $\Omega \backslash \bar{\gamma}_{2}$. In the model below, $\gamma$ corresponds to a thin rigid inclusion located simultaneously on the boundary and inside the domain $\Omega$.

Introduce a set of admissible displacements

$$
K_{b}=\left\{v=\left(v_{1}, v_{2}\right) \in H_{\Gamma_{0}}^{1}\left(\Omega_{\gamma_{2}}\right) \mid[v] \nu \geq 0 \text { on } \gamma_{2},\left.v\right|_{\gamma^{+}} \in R(\gamma)\right\} .
$$




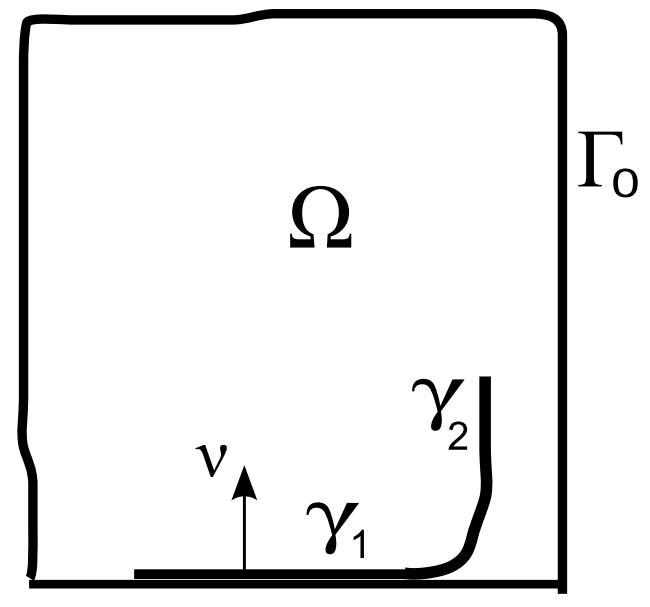

FIG. 6

The equilibrium problem for the elastic body $\Omega_{\gamma_{2}}$ with the inclusion $\gamma$ is as follows. We have to find functions $u=\left(u_{1}, u_{2}\right), \rho_{0} \in R(\gamma), \sigma=\left\{\sigma_{i j}\right\}, i, j=1,2$, such that

$$
\begin{array}{r}
-\operatorname{div} \sigma=f \quad \text { in } \quad \Omega_{\gamma_{2}}, \\
\sigma-A \varepsilon(u)=0 \quad \text { in } \quad \Omega_{\gamma_{2}}, \\
u=0 \quad \text { on } \quad \Gamma_{0}, \\
u=\rho_{0} \quad \text { on } \gamma^{+},[u] \nu \geq 0 \quad \text { on } \quad \gamma_{2}, \\
-\int_{\gamma_{1}} \sigma \nu \cdot u-\int_{\gamma_{2}}[\sigma \nu \cdot u]=0, \\
-\int_{\gamma_{1}} \sigma \nu \cdot v-\int_{\gamma_{2}}[\sigma \nu \cdot v] \geq 0 \quad \forall v \in K_{b} .
\end{array}
$$

It is possible to provide a variational formulation of the problem (50)-(55). Consider the minimization problem

$$
\inf _{v \in K_{b}}\left\{\frac{1}{2} \int_{\Omega_{\gamma_{2}}} \sigma(v) \varepsilon(v)-\int_{\Omega_{\gamma_{2}}} f v\right\}
$$

The problem (56) has (a unique) solution satisfying the variational inequality

$$
u \in K_{b}, \quad \int_{\Omega_{\gamma_{2}}} \sigma(u) \varepsilon(\bar{u}-u) \geq \int_{\Omega_{\gamma_{2}}} f(\bar{u}-u) \quad \forall \bar{u} \in K_{b} .
$$

This statement on the solution existence will be proved later. The difficulty of the proof consists in the absence of a coercivity of the functional in (56) because the boundary of the domain $\Omega_{\gamma_{2}}$ is not smooth, and hence, we cannot use Korn's inequality. Now we check that problem formulations (50)-(55) and (57) are equivalent provided that the solution $u$ is quite smooth. From (57) we obtain

$$
\int_{\Omega_{\gamma_{2}}} \sigma(u) \varepsilon(u)=\int_{\Omega_{\gamma_{2}}} f u
$$


Integration by parts in (58) implies (54). From (57), (58) it follows that

$$
\int_{\Omega_{\gamma_{2}}} \sigma(u) \varepsilon(\tilde{u}) \geq \int_{\Omega_{\gamma_{2}}} f(\tilde{u}) \quad \forall \tilde{u} \in K_{b} .
$$

Integration by parts here implies (55). Hence all relations (50)-(55) can be derived from (57). Conversely, let $\bar{u} \in K_{b}$. From (50) it follows that

$$
\int_{\Omega_{\gamma_{2}}}(-\operatorname{div} \sigma-f)(\bar{u}-u)=0 \quad \forall \bar{u} \in K_{b} .
$$

We integrate by parts and get

$$
\begin{array}{r}
\int_{\Omega_{\gamma_{2}}} \sigma(u) \varepsilon(\bar{u}-u)-\int_{\Omega_{\gamma_{2}}} f(\bar{u}-u) \\
+\int_{\gamma_{1}} \sigma(u) \nu \cdot(\bar{u}-u)+\int_{\gamma_{2}}[\sigma(u) \nu \cdot(\bar{u}-u)]=0 .
\end{array}
$$

Meanwhile, relations (54)-(55) provide

$$
\int_{\gamma_{1}} \sigma(u) \nu \cdot(\bar{u}-u)+\int_{\gamma_{2}}[\sigma(u) \nu \cdot(\bar{u}-u)] \leq 0,
$$

and consequently, (59) implies (57), which is what is needed.

Now we go back to the variational inequality (57) and prove its solvability. We shall use a fictitious domain approach. Consider a fictitious domain $\Omega_{1}$ located as depicted in Fig. 7. Denote by $\Sigma$ a part of the common boundary of domains $\Omega$ and $\Omega_{1}$ (dotted line in Fig. 7) and introduce an extended domain $\tilde{\Omega}_{\gamma}=\left(\Omega \cup \Omega_{1} \cup \Sigma\right) \backslash \bar{\gamma}$. Hence the domain $\tilde{\Omega}_{\gamma}$ has a crack (cut) $\gamma$. We denote by $\tilde{\Gamma}$ an external boundary of $\tilde{\Omega}_{\gamma}$, i.e. $\tilde{\Gamma}=\left(\partial \tilde{\Omega}_{\gamma}\right) \backslash \gamma^{ \pm}$. Now introduce the elasticity tensor $A^{\lambda}=\left\{a_{i j k l}^{\lambda}\right\}$ with a positive parameter $\lambda$,

$$
a_{i j k l}^{\lambda}=\left\{\begin{array}{ccc}
a_{i j k l} & \text { in } & \Omega, \\
\lambda^{-1} a_{i j k l} & \text { in } & \Omega_{1} .
\end{array}\right.
$$

In the extended domain $\tilde{\Omega}_{\gamma}$ we consider a family of boundary value problems. Find functions $u^{\lambda}=\left(u_{1}^{\lambda}, u_{2}^{\lambda}\right), \rho_{0}^{\lambda} \in R(\gamma), \sigma^{\lambda}=\left\{\sigma_{i j}^{\lambda}\right\}, i, j=1,2$, such that

$$
\begin{array}{r}
-\operatorname{div} \sigma^{\lambda}=f \quad \text { in } \quad \tilde{\Omega}_{\gamma}, \\
\sigma^{\lambda}-A^{\lambda} \varepsilon\left(u^{\lambda}\right)=0 \quad \text { in } \quad \tilde{\Omega}_{\gamma}, \\
u^{\lambda}=0 \quad \text { on } \quad \tilde{\Gamma}, \sigma^{\lambda} \nu=0 \quad \text { on } \quad \gamma_{1}^{-}, \\
{\left[u^{\lambda}\right] \nu^{\lambda} \geq 0 \quad \text { on } \quad \gamma_{2}, u^{\lambda}=\rho_{0}^{\lambda} \quad \text { on } \quad \gamma^{+},} \\
\int_{\gamma}\left[\sigma^{\lambda} \nu \cdot u^{\lambda}\right]=0, \\
\int_{\gamma}\left[\sigma^{\lambda} \nu \cdot v\right] \leq 0 \quad \forall v \in K_{g},
\end{array}
$$

where the set of admissible displacements is defined as follows:

$$
K_{g}=\left\{v=\left(v_{1}, v_{2}\right) \in H_{\tilde{\Gamma}}^{1}\left(\tilde{\Omega}_{\gamma}\right) \mid[v] \nu \geq 0 \text { on } \gamma_{2},\left.v\right|_{\gamma^{+}} \in R(\gamma)\right\} .
$$




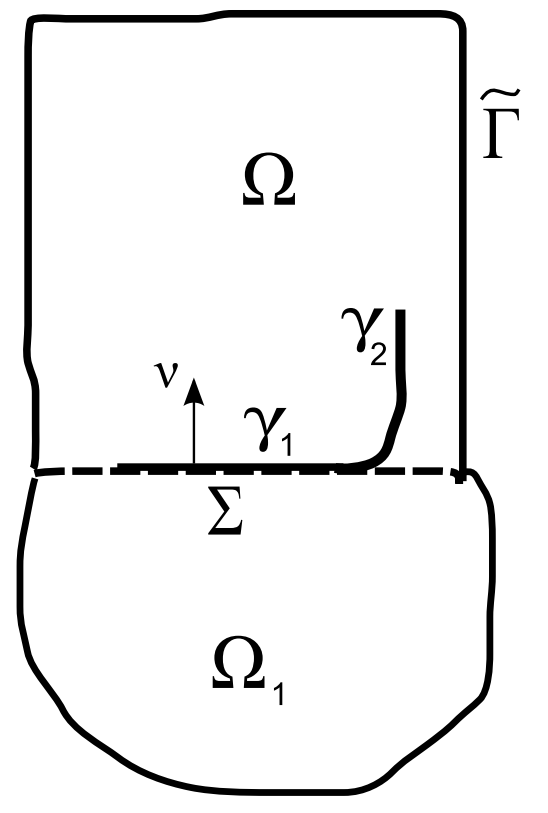

FIG. 7

The problem (61)-(66) for each fixed $\lambda$ describes an equilibrium of the body $\tilde{\Omega}_{\gamma}$ with the thin rigid inclusion $\gamma$. Note that for this problem a delamination takes place on $\gamma^{-}$.

The problem (61) -(66) is solvable. Indeed, consider the minimization problem

$$
\inf _{v \in K_{g}}\left\{\frac{1}{2} \int_{\tilde{\Omega}_{\gamma}} \sigma^{\lambda}(v) \varepsilon(v)-\int_{\tilde{\Omega}_{\gamma}} f v\right\}
$$

This problem has a solution satisfying the variational inequality

$$
u^{\lambda} \in K_{g}, \quad \int_{\tilde{\Omega}_{\gamma}} \sigma^{\lambda}\left(u^{\lambda}\right) \varepsilon\left(\bar{u}-u^{\lambda}\right) \geq \int_{\tilde{\Omega}_{\gamma}} f\left(\bar{u}-u^{\lambda}\right) \quad \forall \bar{u} \in K_{g} .
$$

From (67) it follows that

$$
\int_{\tilde{\Omega}_{\gamma}} \sigma^{\lambda}\left(u^{\lambda}\right) \varepsilon\left(u^{\lambda}\right)=\int_{\tilde{\Omega}_{\gamma}} f u^{\lambda} .
$$

Consequently, we have uniformly in $\lambda \in\left(0, \lambda_{0}\right)$,

$$
\left\|u^{\lambda}\right\|_{H_{\tilde{\Gamma}}^{1}\left(\tilde{\Omega}_{\gamma}\right)} \leq c,\left\|u^{\lambda}\right\|_{H^{1}\left(\Omega_{1}\right)}^{2} \leq c \lambda .
$$

Choosing a subsequence we can assume that as $\lambda \rightarrow 0$,

$$
u^{\lambda} \rightarrow u \text { weakly in } H_{\tilde{\Gamma}}^{1}\left(\tilde{\Omega}_{\gamma}\right) ; u^{\lambda} \rightarrow 0 \text { strongly in } H^{1}\left(\Omega_{1}\right) .
$$

We now take $\bar{u} \in K_{g}$ such that $\bar{u}=0$ in $\Omega_{1}$. Note that a restriction of this function $\bar{u}$ to $\Omega_{\gamma_{2}}$ belongs to $K_{b}$. Substituting $\bar{u}$ in (67) we can pass to the limit as $\lambda \rightarrow 0$. The limiting variational inequality has the form

$$
u \in K_{b}, \quad \int_{\Omega_{\gamma_{2}}} \sigma(u) \varepsilon(\bar{u}-u) \geq \int_{\Omega_{\gamma_{2}}} f(\bar{u}-u) .
$$


Since a restriction $\left.\bar{u}\right|_{\Omega_{\gamma_{2}}}$ can be taken as an arbitrary element of the set $K_{b}$ we arrive at (57). This proves a solution existence of (57).

Acknowledgement. The work was supported by the Russian Foundation for Basic Research (N 10-01-00054), and the grant of the Siberian Branch of RAS (N 90).

\section{REFERENCES}

[1] Fichera G. Boundary value problems of elasticity with unilateral constraints. In: Handbuch der Physik, Band 6a/2, Springer-Verlag, 1972.

[2] Khludnev A.M., Leugering G. On elastic bodies with thin rigid inclusions and cracks, Math. Meth. Appl. Sciences, 2010, v. 33, N16, pp. 1955-1967. MR2744613

[3] Khludnev A.M., Kovtunenko V.A. Analysis of cracks in solids. Southampton-Boston, WIT Press, 2000.

[4] Khludnev A.M., Tani A. Unilateral contact problems for two inclined elastic bodies. European Journal of Mechanics A/Solids, 2008, v.27, N3, pp. 365 - 377. MR2407924 (2009d:74066)

[5] Khludnev A.M., Novotny A.A., Sokolowski J., Zochowski A. Shape and topology sensitivity analysis for cracks in elastic bodies on boundaries of rigid inclusions. Journal of the Mechanics and Physics of Solids, 2009, v. 57, N 10, pp. 1718-1732. MR2567570

[6] Khludnev A.M., Sokolowski J. Modelling and control in solid mechanics. Basel-Boston-Berlin, Birkhäuser, 1997. MR1433133 (98c:93004)

[7] Khludnev A.M., Leugering G. On the equilibrium of elastic bodies with thin rigid inclusions. Doklady Physics, 2010, v.430, N1, pp. 47-50. MR.2668827

[8] Khludnev A.M., Sokolowski J. Smooth domain method for crack problems. Quart. Appl. Math., 2004, v. 62, N3, pp. 401-422. MR2086037 (2005d:35096)

[9] Khludnev A.M., Tani A. Overlapping domain problems in the crack theory with possible contact between crack faces. Quart. Appl. Math., 2008, v. 66, N 3, pp. 423-435. MR.2445521(2009i:74084)

[10] Kovtunenko V.A. Invariant integrals in nonlinear problem for a crack with possible contact between crack faces. J. Appl. Math. Mech., 2003, v. 67, N. 1, pp. 109-123. MR.1997626 (2004e:74074)

[11] Mallick P. K. Fiber-reinforced composites. Materials, manufacturing, and design, Marcel Dekker, Inc., 1993.

[12] Neustroeva N.V. Contact problem for elastic bodies of different dimensions. Vestnik of Novosibirsk State University (math., mech., informatics), 2008, v. 8, N4, pp. 60-75.

[13] Neustroeva N.V. Unilateral contact of elastic plates with a rigid inclusion. Vestnik of Novosibirsk State University (math., mech., informatics), 2009, N4, pp. 51-64.

[14] Prechtel M., Leugering G., Steinmann P., Stingl M. Towards optimization of crack resistance of composite materials by adjusting of fiber shapes, Engineering fracture mechanics, 2011, v.78, N 6, pp. 944-960.

[15] Rudoŭ E.M. Differentiation of energy functionals in the problem of a curvilinear crack with possible contact between crack faces. Izvestiya RAN, Solid mechanics, 2007, N 6, pp. 113-127.

[16] Rudoŭ E.M. Griffith formula and Rice-Cherepanov integral for a plate with a rigid inclusion. Vestnik of Novosibirsk State University (math., mech., informatics), 2010, v.10, N 2, pp. 98-117.

[17] Rudoŭ E.M. Asymptotic behavior of energy functional for a three dimensional body with a rigid inclusion and a crack. J. Appl. Mech. Techn. Phys., 2011, v.52, N 2, pp. 252-263.

[18] Rudor E.M. Asymptotics of energy functional for an elastic body with a rigid inclusion. 2D problem. J. Appl. Math. Mech., 2011, v. 75, N 5, pp. 719-729.

[19] Rotanova T.A. Unilateral contact problem for two plates with a rigid inclusion. Vestnik of Novosibirsk State University (math., mech., informatics), 2011, v. 11, N 1, pp. 87-98. 